\title{
Are lateral ankle injuries in children fractures or sprains?
}

\author{
Reviewed by: Erica Beatty*; Maude Deschênes*; Patrick Archambault ${ }^{\dagger \ddagger}$
}

Clinical question

What is the prevalence of Salter-Harris type I distal fibula fractures in children with lateral ankle injuries?

Article chosen

Boutis K, Plint A, Stimec J, et al. Radiograph-negative lateral ankle injuries in children occult growth plate fracture or sprain? JAMA Pediatr 2016;170(1):e154114.

$\overline{\text { Keywords: pediatric, Salter-Harris type I fractures, ankle sprains }}$

\section{BACKGROUND AND OBJECTIVES}

With regard to musculoskeletal injuries in children, traditional medical teachings insist that growth plate injuries are more common than ligamentous injuries because of the inherent weakness of the epiphyseal cartilage. These teachings are based on a study by Salter and Harris published in 1963. Therefore, children with traumatic injuries and negative radiographs are presumed to have a Salter-Harris type I fracture and are treated accordingly with three to six weeks of immobilization. This study aimed to confirm the prevalence of Salter-Harris type I distal fibula fractures (SH1DF) and to compare functional recovery between children with magnetic resonance imaging (MRI)-identified fractures and ligamentous injuries, if treated with a removable ankle stirrup to reduce overtreatment.

\section{POPULATION STUDIED}

The study population included children aged 5 to 12 years who presented to the emergency department
(ED) with a clinically suspected SH1DF. A SH1DF was suspected if there was an isolated lateral ankle injury with negative radiographs. Exclusion criteria included an ankle fracture within the last three months, a preexisting musculoskeletal disease, or a coagulopathy, as well as any patients presenting after 72 hours.

\section{STUDY DESIGN}

This was a prospective cohort study evaluating the proportion of SH1DF in children with lateral ankle injuries and negative radiographs. Secondary outcomes included a functional score at one month, namely the Activity Scale for Kids (ASKp), as well as return to full weight-bearing and activities at three months.

In total, 271 children aged 5 to 12 years were screened, of whom 140 were eligible, with consent to participate. All but five children had an MRI of both the injured and contralateral ankle within seven days of the injury. Specific radiologic diagnostic criteria were established in advance, and all imagery was reviewed by three radiologists, all of whom were blinded. All radiologists were practising at a pediatric tertiary care centre; two of the three radiologists were specialists of musculoskeletal radiology. All patients were treated with a removable air-stirrup ankle brace with a selfdirected return to activities. Follow-up included an ASKp questionnaire one month post-injury completed by the parents, an orthopaedic follow-up and repeat ankle radiographs at one month, and a telephone follow-up evaluating weight-bearing and the return to usual activities at three months.

\footnotetext{
From the *Département de médecine d'urgence et médecine familiale, Faculté de médecine, Université Laval, Québec, QC; †Centre intégré de santé et de services sociaux de Chaudière-Appalaches (Centre hospitalier affilié universitaire de l'Hôtel-Dieu de Lévis), Lévis, QC; and the ¥Division de soins intensifs, Département d'anesthésie, Université Laval, Québec, QC.
}

Correspondence to: Dr. Patrick Archambault, 143 rue Wolfe, Lévis, QC G6V 3Z1, Canada; Email: patrick.m.archambault@gmail.com 


\section{RESULTS}

Of the 135 children with a diagnostic MRI for a lateral ankle injury, only 4 (3\%) had a confirmed SH1DF.

Ligamentous injuries were found in 108 (80\%) of the patients, and $73(68 \%)$ were intermediate- to highgrade ligamentous injuries.

Distal fibular avulsion fractures were identified in $38(28 \%)$ of the patients, and bony contusions were identified in $27(22 \%)$ of the patients.

There were no detectable differences in the ASKp score or functional recovery between children with SH1DF and children with ligamentous injuries of any grade at one and three months. More precisely, at one month post-injury, the ASKp scores were 91 in the SH1DF group, 82 in the high-grade ligament injury group, and 85 in the low-grade ligament injury group. At the 3-month follow-up, 100\% of the children with fractures were able to return to activities "almost all of the time," as compared with 96\% of children with no fractures.

\section{CONCLUSION}

Most children with lateral ankle injuries and no fracture on radiographs had ligamentous sprain injuries. If a fracture was indeed present on MRI, it was likely to be an avulsion fracture, not a Salter-Harris type I fracture. The prevalence of SH1DF was 3\%. Furthermore, if treated with a removable ankle brace and self-regulated return to activities, children with fractures, an avulsion or another type of fracture, had comparable recovery when compared with those who had sprain injuries.

\section{COMMENTARY}

This was a well-designed prospective cohort study. The study team did an excellent job in limiting biases, including having all imagery reviewed by three radiologists and using the patients' uninjured ankle as a control. All follow-ups, except those with the orthopaedic surgeon, were also blinded. There was a potential for selection bias, as patients were recruited between 8 a.m. and 11 p.m. only. Nevertheless, this bias might have been mitigated by the fact that children with musculoskeletal injuries generally present during daytime hours.

Overall, the study was adequately powered to determine a $3 \%$ prevalence of Salter-Harris type I fractures, which it did indeed confirm. Unfortunately, as much as the results may hint at a difference in functional recovery that favoured patients with fractures, the study was not appropriately designed or adequately powered to detect said difference; the sample size was not calculated for the secondary outcomes. Therefore, it is difficult to draw definitive conclusions as to functional recovery. However, this was not the first study to evaluate functional recovery of lateral ankle injuries with less aggressive treatments. Previous studies on less invasive treatments for lateral ankle injuries, including SH1DF, show equal or improved outcomes at four weeks, with stirrup braces or compressive bandages (Tubigrip) over casting. ${ }^{1,2}$ Unfortunately, none of these studies, including the one being reviewed here, evaluate the long-term disability associated with possible growth arrest.

Although not evaluated by this paper, previous work by Boutis et al. also determined that the treatment of lateral ankle fractures with a stirrup brace reduced both health care costs and parental work loss versus traditional casting. ${ }^{1}$

\section{CONCLUSIONS}

This study confirms that SH1DF are actually much less common than ankle sprains in children, contrary to traditional medical teachings. It also provides additional evidence that less aggressive treatment of lateral ankle injuries, more precisely an ankle stirrup and self-directed return to activities, may provide adequate functional recovery. Unfortunately, additional studies evaluating long-term functional recovery with a removable ankle stirrup over casting in SH1DF are necessary to clarify the long-term safety given the possibility of growth arrest.

\section{CLINICAL BOTTOM LINE}

For children between the ages of 5 and 12 years who present to the ED with radiograph-negative lateral ankle injuries, the most likely diagnosis is a sprain. In light of this study, one may consider treating these patients with a removable ankle stirrup and return to activities as tolerated. However, as the long-term safety of this treatment has not been established, shared decision-making with parents ${ }^{3,4,5}$ as to the risks and benefits of a stirrup brace versus casting may be appropriate to determine the definitive treatment.

Acknowledgements: The authors would like to thank Maude Dionne, research coordinator, who helped prepare and review the manuscript. EB and MD analyzed the article. EB was responsible for the English written version, and $\mathrm{MD}$ was 
responsible for the French written version. PA supervised the critical appraisal and writing of the manuscript. All authors had the opportunity to read and review the final manuscript before submission and provided their permission to publish the article.

Competing interests: No financing was accepted by the authors or members of the team to produce this article. The authors have no conflicts of interest to declare.

\section{REFERENCES}

1. Boutis K, Willan AR, Babyn P, et al. A randomized, controlled trial of a removable brac versus casting in children with low-risk ankle fractures. Pediatrics 2007;119(6):1256-63.
2. Gleeson AP, Stuart MJ, Wilson B, Phillips B. Ultrasound assessment and conservative management of inversion injuries of the ankle in children: plaster of Paris versus Tubigrip. 7 Bone foint Surg Br 1996;78(3): 484-7.

3. Kunneman M, Montori VM, Castaneda-Guarderas A, Hess EP. What is shared decision making? (and what it is not). Acad Emerg Med 2016;23(12):1320-4.

4. O'Connor AM. Using deciion aids to help patients navigate the "grey zone" of medical decision-making. CMAf 2007;176(11):1597-8.

5. Légaré F, Stacey D, Forest PG, et al. Milestones, barriers and beacons: shared decision making in Canada inches ahead. Z Evid Fortbild Qual Gesundhwes 2017; $123-4: 23-7$ 